\title{
A NOTE ON THE HOLOMORPHIC FUNCTIONAL CALCULUS IN A BANACH ALGEBRA
}

\author{
G. R. ALLAN
}

1. Introduction. The holomorphic functional calculus for functions of several complex variables has been through a number of stages both with regard to the detailed content of the main theorem and also its method of proof. (See in particular [5], [2], [7], [1].) A good recent exposition, using a method due essentially to Waelbroeck, is contained in [3]. A different approach, which we feel to be the most illuminating and which also originates with Waelbroeck (in [7]), is expounded, for example, in Chapter III of Hörmander's book [5] (and also in [4]). An apparent disadvantage of this treatment, in comparison with that of [3], is that the functional calculus map is not shown to be a homomorphism. This deficiency can be made good, as was done in [7], by using deep results of Cartan concerning the ideal theory of holomorphic functions, but this destroys the essential elementarity of the treatment in [5], which uses very little machinery. The point of the present note is to give an elementary proof of a crucial lemma (Lemma 2), in the spirit of the methods of Chapters II and III of [5] which then leads to a very simple deduction of the full result as stated in [3, Chapitre I $\S 4$, Théorème 1$]$. Lemma 2 is the only result for which we give a detailed proof, but it seemed desirable to give a brief indication of the relevance of this lemma in setting up the functional calculus and we accordingly outline the main steps.

2. A lemma on certain ideals of holomorphic functions. We state first, without proof, the fundamental result on the extension of holomorphic functions defined on submanifolds, in the special case needed. We prefer to use the 'open set form' rather than that of Theorem 2.7.6 of [5]. An elementary proof may easily be given based on, say, Theorem 2.7.8 of [5]. If $W$ is an open polydisc in $C^{n}$ and if $Q_{1}, \cdots Q_{l}$ are polynomials, a set such as

$$
U=\left\{z \in W:\left|Q_{j}(z)\right|<1(j=1, \cdots, l)\right\}
$$

will be called a polynomial polyhedron. (The case $U=W$ will be included, corresponding to $l=0$.)

Lemma 1 (Extension Theorem). Let $U$ be a polynomial polyhedron in $C^{n}$, let $P_{1}, \cdots, P_{k}$ be polynomials on $C^{n}$ and let $U_{P}$ be the polynomial polyhedron

Received by the editors August 19, 1968. 


$$
U_{P}=\left\{z \in U:\left|P_{j}(z)\right|<1(j=1, \cdots, k)\right\} .
$$

Let $D$ be the open unit disc in $C$, let $V=U \times D^{k}$ and let $\mu$ denote the 'Oka map' from $\mathrm{C}^{n}$ into $\mathrm{C}^{n+k}$ defined by

$$
\mu(z)=\left(z, P_{1}(z), \cdots, P_{k}(z)\right) \quad\left(z \in C^{n}\right) .
$$

Then for any holomorphic function $f$ on $U_{P}$ there is a holomorphic function $F$ on $V$ such that

$$
F(\mu(z))=f(z) \quad\left(z \in U_{P}\right) .
$$

Thus Lemma 1 states that the mapping $F \rightarrow F \circ \mu$ is a homomorphism of $H(V)$ onto $H\left(U_{P}\right)$ (where, for any open subset $G$ of $C^{r}, H(G)$ is the algebra of holomorphic functions on $G$ ). The aim of Lemma 2 is to specify the kernel of this homomorphism. As stated in the introduction, the elementary proof of Lemma 2 is the point of the paper.

Lemma 2. Let $U, P_{1}, \cdots, P_{k}, U_{P}$ and $\mu$ be as in Lemma 1. Let $F$ be a holomorphic function on $V \equiv U \times D^{k}$ such that $F(\mu(z))=0$ for $z \in U_{P}$. Then there exist holomorphic functions $H_{1}, \cdots, H_{k}$ on $V$ such that (writing $\left.w=\left(w_{1}, \cdots, w_{k}\right) \in C^{k}\right)$,

$$
F(z, w)=\sum_{j=1}^{k} H_{j}(z, w)\left(w_{j}-P_{j}(z)\right) \quad((z, w) \in V) .
$$

Proof. The proof will be by induction on $k$.

Case $k=1$. This is essentially trivial. For each point $\left(z_{0}, w_{0}\right) \in V$ we choose an open polydisc $D$ contained in $V$ with centre $\left(z_{0}, w_{0}\right)$ and a holomorphic function $H$ on $D$ such that $F(z, w)=H(z, w)(w-P(z))$ on $D$. (Recall that $w \in C$ since $k=1$.) Indeed if $\left(z_{0}, w_{0}\right) \notin M=$ range $\mu$ we may then choose $D$ such that $D \cap M=\varnothing$ and the corresponding $H$ is necessarily just $F(z, w) /(w-P(z))$ on $D$. If, on the other hand, $\left(z_{0}, w_{0}\right) \in M$, so that $w_{0}=P\left(z_{0}\right)$, the existence of a suitable $H$ follows by considering a power series expansion in the variables $z-z_{0}$, $w-P(z)$.

If $D, D^{\prime}$ are two such polydiscs and $H, H^{\prime}$ are the corresponding functions then $H$ and $H^{\prime}$ certainly agree on $\left(D \cap D^{\prime}\right) \backslash M$ and hence on $D \cap D^{\prime}$.

Thus we have defined a holomorphic function $H$ on the whole of $V$ such that $F(z, w)=H(z, w)(w-P(z))$ on $V$. This completes the proof for the case $k=1$.

Case $k>1$. We make the inductive assumption that the theorem is true for less than $k$ polynomials $P_{\boldsymbol{j}}$. 
Define $U_{1}=\left\{z \in U:\left|P_{k}(z)\right|<1\right\}$, so that $U_{1}$ is also a polynomial polyhedron in $\mathbf{C}^{n}$.

Define $\mu_{1}: C^{n} \rightarrow C^{n+k-1}$ by

$$
\mu_{1}(z)=\left(z, P_{1}(z), \cdots, P_{k-1}(z)\right),
$$

and $\mu_{2}: \mathbf{C}^{n+k-1} \rightarrow \mathbf{C}^{n+k}$ by

$$
\mu_{2}\left(z, w_{1}, \cdots, w_{k-1}\right)=\left(z, w_{1}, \cdots, w_{k-1}, P_{k}(z)\right) .
$$

Clearly $\mu=\mu_{2} \circ \mu_{1}$.

Then $\mu_{2}\left(U_{1} \times D^{k-1}\right) \subseteq V$ and we can thus define the holomorphic function $G=F \circ \mu_{2}$ on $U_{1} \times D^{k-1}$. Then, for $z \in U_{1}$,

$$
G\left(\mu_{1}(z)\right)=\left(F \circ \mu_{2}\right)\left(\mu_{1}(z)\right)=F(\mu(z))=0,
$$

and so, by the inductive hypothesis, there exist holomorphic functions $G_{1}, \cdots, G_{k-1}$ on $U_{1} \times D^{k-1}$ such that

$$
\begin{aligned}
F\left(z, w_{1}, \cdots, w_{k-1}, P_{k}(z)\right) & =G\left(z, w_{1}, \cdots, w_{k-1}\right) \\
& =\sum_{j=1}^{k-1} G_{j}\left(z, w_{1}, \cdots, w_{k-1}\right)\left(w_{j}-P_{j}(z)\right)
\end{aligned}
$$

for $\left(z, w_{1}, \cdots, w_{k-1}\right) \in U_{1} \times D^{k-1}$.

But now, since we may write

$$
U_{1} \times D^{k-1}=\left\{\left(z, w_{1}, \cdots, w_{k-1}\right) \in U \times D^{k-1}:\left|P_{k}(z)\right|<1\right\},
$$

an application of Lemma 1 yields the existence of holomorphic functions $H_{1}, \cdots, H_{k-1}$ on $U \times D^{k}$ such that

$$
\begin{aligned}
H_{j}\left(z, w_{1}, \cdots, w_{k-1}, P_{k}(z)\right) & =H_{j}\left(\mu_{2}\left(z, w_{1}, \cdots, w_{k-1}\right)\right) \\
& =G_{j}\left(z, w_{1}, \cdots, w_{k-1}\right)
\end{aligned}
$$

for $\left(z, w_{1}, \cdots, w_{k-1}\right) \in U_{1} \times D^{k-1}$.

Now define $H$ on $V=U \times D^{k}$ by

$$
H(z, w)=F(z, w)-\sum_{j=1}^{k-1} H_{j}(z, w)\left(w_{j}-P_{j}(z)\right),
$$

where $w=\left(w_{1}, \cdots, w_{k}\right)$. Then $H$ is holomorphic on $V$ and, for $\left(z, w_{1}, \cdots, w_{k-1}\right) \in U_{1} \times D^{k-1}, H\left(\mu_{2}\left(z, w_{1}, \cdots, w_{k-1}\right)\right)=0$. Hence, by the case $k=1$, already proved, there exists a holomorphic function $H_{k}$ on $V$ such that

$$
H(z, w)=H_{k}(z, w)\left(w_{k}-P_{k}(z)\right) \quad((z, w) \in V) .
$$

\section{Thus}




$$
F(z, w)=\sum_{j=1}^{k} H_{j}(z, w)\left(w_{j}-P_{j}(z)\right) \quad((z, w) \in V)
$$

and the lemma is proved.

3. Application to holomorphic functional calculus. Let $A$ be a commutative complex Banach algebra with identity $e$ and let $x_{1}$, $\cdots, x_{n} \in A$. The holomorphic functional calculus theorem yields a continuous homomorphism $\Theta_{\left(x_{1}, \cdots, x_{n}\right)}$ from the algebra of germs of holomorphic functions on the joint spectrum $\sigma_{A}\left(x_{1}, \cdots, x_{n}\right)$ into $A$ itself. This homomorphism takes the $j$ th coordinate projection to the element $x_{j}$ of $A$. The set of all these homomorphisms $\Theta_{\left(x_{1}, \cdots, x_{n}\right)}$ for all $n$-tuples of elements of $A$ is unique subject to a natural consistency requirement. We refer the reader to $[3$, Chapitre $I, \S 4$, Théorème 1$]$ for a complete statement.

The well-known method of Arens-Calderón [2] enables reduction of the problem to the case of Silov [6] in which $\left\{x_{1}, \cdots, x_{n}\right\}$ are a set of generators for $A$. In order to indicate briefly the role of Lemma 2 we give an indication of the proof of the following proposition. The deduction of the full result, as given in [3], is then quite a simple matter involving no essentially new point.

Proposition. Let $\left\{x_{1}, \cdots, x_{n}\right\}$ generate $A$, let $W$ be an open neighbourhood of $\sigma_{A}\left(x_{1}, \cdots, x_{n}\right)$ in $C^{n}$ and let $H(W)$ denote the algebra of complex-valued holomorphic functions on $W$ topologized by uniform convergence on compact subsets. Then there is a continuous homomorphism $\theta$ from $H(W)$ into $A$ such that $\theta(1)=e, \theta\left(z_{j}\right)=x_{j}(j=1, \cdots, n)$. (Here 1 denotes the unit function in $H(W)$ and $z_{j}$ denotes the $j$ th coordinate map.)

OUtLINe of Proof. It is a simple matter to choose polynomials $P_{1}, \cdots, P_{k}$ on $C^{n}$ and an open polydisc $U$ such that $\sigma_{A}\left(x_{1}, \cdots, x_{n}\right) \subseteq U_{P} \equiv\left\{z \in U:\left|P_{j}(z)\right|<1 \quad(j=1, \cdots, n)\right\} \subseteq W$

and we thus may suppose without loss of generality that $W=U_{P}$. (See any of the several accounts of the functional calculus already referred to.)

Let $D$ be the open unit disc in $C$ and let $V=U \times D^{k}$. Then Lemma 1 gives a continuous homomorphism $F \rightarrow F \circ \mu$ of $H(V)$ onto $H\left(U_{P}\right)$. Call this homomorphism $\mu^{*}$. If we define $y_{j}=P_{j}\left(x_{1}, \cdots, x_{n}\right)$ $(j=1, \cdots, k)$ then $\sigma_{A}\left(x_{1}, \cdots, x_{n}, y_{1}, \cdots, y_{k}\right)=\mu\left(\sigma_{A}\left(x_{1}, \cdots, x_{n}\right)\right)$ $\subset V$. Since $V$ is a polydisc it is a purely elementary matter to define a (unique) continuous homomorphism $\theta_{0}: H(V) \rightarrow A$ such that 
$\theta_{0}(1)=e, \theta_{0}\left(z_{j}\right)=x_{j}(j=1, \cdots, n), \theta_{0}\left(z_{n+j}\right)=y_{j}(j=1, \cdots, k)$. In order to deduce the existence of a (unique) homomorphism $\theta: H\left(U_{P}\right)$ $\rightarrow A$ such that $\theta \circ \mu^{*}=\theta_{0}$ it is now merely necessary to show that ker $\mu^{*} \subseteq \operatorname{ker} \theta_{0}$. An element of $H(V)$ such as $z_{n+j}-P_{j}\left(z_{1}, \cdots, z_{n}\right)$ $(j=1, \cdots, k)$ is certainly in $\operatorname{ker} \theta_{0}$ since

$$
\theta_{0}\left(z_{n+j}-P_{j}\left(z_{1}, \cdots, z_{n}\right)\right)=y_{j}-P_{j}\left(x_{1}, \cdots, x_{n}\right)=0 .
$$

But, by Lemma 2 , ker $\mu^{*}$ is generated by the elements $z_{n+j}$ $-P_{j}\left(z_{1}, \cdots, z_{n}\right)(j=1, \cdots, k)$ and thus $\operatorname{ker} \mu^{*} \subseteq \operatorname{ker} \theta_{0}$. This proves the existence of $\theta$. To prove the continuity of $\theta$ we observe that both $H(V)$ and $H\left(U_{P}\right)$ are Fréchet spaces and that $\mu^{*}$ is a continuous homomorphism of $H(V)$ onto $H\left(U_{P}\right)$. Thus any null sequence in $H\left(U_{P}\right)$ lifts to a null sequence in $H(V)$ and the continuity of $\theta$ may be deduced from that of $\theta_{0}$.

\section{REFERENCES}

1. R. Arens, The analytic functional calculus in commutative topological algebras, Pacific J. Math. 11 (1961), 405-429.

2. R. Arens and A. P. Calderon, Analytic functions of several Banach algebra elements, Ann. of Math. 62 (1955), 204-16.

3. N. Bourbaki, Théories spectrales, Hermann, Paris, 1967.

4. R. C. Gunning and H. Rossi, Analytic functions of several complex variables, Prentice-Hall, Englewood Cliffs, N. J., 1965.

5. L. Hörmander, An introduction to complex analysis in several variables, Van Nostrand, New York, 1966.

6. G. E. Silov, On the decomposition of a commutative normed ring into a direct sum of ideals, Mat. Sb. 32 (74) (1953), 353-364; Amer. Math. Soc. Transl. (2) 1 (1955), 37-48.

7. L. Waelbroeck, Le calcul symboliques dans les algebres commutatives, J. Math. Pures Appl. 33 (1954), 147-186.

The University, Newcastle upon Tyne, England 